\title{
Pair transfer processes probed at deep sub barrier energies
}

\author{
L. Corradi ${ }^{1, a}$, S. Szilner ${ }^{4}$, G. Pollarolo ${ }^{3}$, P. Mason ${ }^{1}$, E. Farnea ${ }^{2}$, E. Fioretto ${ }^{1}$, A. Gadea ${ }^{5}$, F. Haas ${ }^{6}$, D. Jelavić-Malenica ${ }^{4}$ \\ N. Mărginean ${ }^{7}$, C. Michelagnoli ${ }^{1}$, G. Montagnoli ${ }^{2}$, D. Montanari ${ }^{2}$, F. Scarlassara ${ }^{2}$, N. Soić ${ }^{4}$, A. M. Stefanini ${ }^{1}$, C. A. Ur ${ }^{2}$, \\ and J. J. Valiente-Dobón ${ }^{1}$ \\ 1 Istituto Nazionale di Fisica Nucleare, Laboratori Nazionali di Legnaro, I-35020 Legnaro, Italy \\ 2 Dipartimento di Fisica, Università di Padova, and Istituto Nazionale di Fisica Nucleare, I-35131 Padova, Italy \\ 3 Dipartimento di Fisica Teorica, Università di Torino, and Istituto Nazionale di Fisica Nucleare, 10125 Torino, Italy \\ 4 Ruđer Bošković Institute, HR-10 001 Zagreb, Croatia \\ 5 IFIC, CSIC-Universidad de Valencia, Valencia, Spain \\ 6 Institut Pluridisciplinaire Hubert Curien, CNRS-IN2P3, Université de Strasbourg, F-67037 Strasbourg, France \\ 7 Horia Hulubei National Institute of Physics and Nuclear Engineering, 077125, Bucharest-Magurele, Romania
}

\begin{abstract}
Multinucleon transfer cross sections in the system ${ }^{40} \mathrm{Ca}+{ }^{96} \mathrm{Zr}$ have been measured at bombarding energies ranging from the Coulomb barrier to $\sim 25 \%$ below. Target-like (lighter) recoils in inverse kinematics have been completely identif ed in A,Z and Q-value with the large solid angle magnetic spectrometer PRISMA. The experimental slopes of the neutron transfer probabilities at large internuclear separation are consistent with the values derived from the binding energies. A phenomenological interpretation of the transfer probabilities indicates the presence of enhanced values for the even number of neutron transfers.
\end{abstract}

\section{Introduction}

In quasi-elastic reactions nuclei enter into contact through the tail of their density distributions and nucleon transfer processes take place in between levels close to the Fermi surfaces of the donor and acceptor. At large distances between the centers of the interacting nuclei the reaction mechanism conditions are much simplif ed compared to those near the strong absorption radius. Nuclei are only slightly inf uenced by the nuclear potential and follow almost pure Coulomb trajectories. Excitation energies are restricted to few $\mathrm{MeV}$ and absorption is reduced to a small contribution. These peculiar conditions should in principle allow to extract more quantitative information on the mechanism of multiple transfer processes, for example on the relative contribution of single particle and more complex degrees of freedom which include nucleon-nucleon correlations [1]. Very scarse studies have been performed so far in reactions with heavy ions, for known experimental and theoretical difficulties.

In the very low energy domain the nuclear couplings are dominated by one particle transfer processes, the corresponding form factors having a decay length of $\sim 1.3 \mathrm{fm}$ which is related to the binding energy of the transferred nucleon. In ${ }^{16} \mathrm{O}+{ }^{208} \mathrm{~Pb}$ [2] it has been shown that the two nucleon transfer is dominated by a successive mechanism. In an analysis [3] which includes the ${ }^{14} \mathrm{C}+{ }^{124} \mathrm{Sn},{ }^{138} \mathrm{Ba}$ systems, it has been discussed how the successive mechanism displays the same type of constructive coherence as the simultaneous transfer and leading to similar enhancements

\footnotetext{
a e-mail: corradi@lnl.infn.it
}

of the calculated cross sections with respect to pure conf gurations. These enhancements are a measure of the sensitivity of two-nucleon transfer reactions to pairing correlations.

One expects that the successive mechanism dominates also in multinucleon transfer processes, in particular in reactions with heavier ions. The analysis of inclusive heavy ion transfer data obtained at energies slightly above the Coulomb barrier [4] evidenced how neutron pick-up channels are reasonably well described by assuming an independent particle transfer mechanism [5-7]. To describe the behaviour of the full experimental isotopic yields including proton transfers one needs to include other degrees of freedom, like pair modes $[8,9]$. These modes, introduced in a macroscopic way [10], seem more required for protons than for neutrons, qualitatively explained by the fact that the one neutron transfer has an order of magnitude larger cross section than the one proton transfer and therefore the possible contribution of a pair mode is likely to be masked in the successive process. The conclusions are based on studies mainly performed close to the grazing angle, which corresponds to interaction distances where absorption is signif cant. Moreover, excitation energies are non negligible and other complex effects, like nucleon evaporation, complicate the transfer mechanism. Thus, we found of great interest to perform a study well below the barrier where, as said before, the conditions are much simplif ed.

Measurements of transfer yields in the same range of distances between the interacting nuclei can be done either via an angular distribution at a f xed bombarding energy or via an excitation function at a f xed angle. The two 
methods should be equivalent provided the Q-value ranges probed in each transfer channel are similar. In direct kinematics transfer at very large distances corresponds to the forward part of the angular distribution, and the overhelming yield of elastically scattered particles often prevents to identify transfer channels, especially isotopes of the projectile. On the other hand, at energies below the barrier measurements of heavy-ion transfer reactions have signif cant technical difficulties and this is why available data are extremely scarse or almost not existing. Angular distributions result, in the center of mass frame, in a strong backward peaking. The absolute yield gets very small, therefore high efficiency is required. At the same time, mass and nuclear charge resolutions must be maintained at a level sufficient to distinguish the different reaction channels. In most cases the projectile has a signif cant fraction of the target mass, leaving the backscattered projectile-like fragment with such a low energy that usual identif cation techniques become invalid. To partly overcome this problem with heavy ions, two methods have been employed so far, one that identif es the (heavy) target recoils at zero degree with recoil mass spectrometers $[11,12]$ and another making use of inverse kinematics, detecting the lighter targetlike particles with a magnetic spectrometer at very forward angles $[13,14]$. One serious limitation of the f rst method is the very low energy resolution of the detected ions, with cross sections obtained integrating a broad Q-value range. With the second method one obtains much better nuclear charge and Q-value resolutions, but the small solid angle of conventional spectrometers limits statistical accuracy at sub-Coulomb energies.

We here discuss part of the the results of a $\mathrm{f}$ rst subbarrier transfer measurement performed in the ${ }^{40} \mathrm{Ca}+{ }^{96} \mathrm{Zr}$ system in inverse kinematics with the PRISMA spectrometer [15] which overcomes the mentioned limitations. Projectile and target are closed or near-closed shell nuclei for both neutrons and protons, thus representing a good reference for a quantitative comparison with theoretical calculations. This experimental and theoretical environment provides very suitable conditions for a proper study of the mechanism of multiple transfer processes.

\section{The experiment}

We measured an excitation function making use of a ${ }^{96} \mathrm{Zr}$ beam delivered from the XTU-Tandem + ALPI accelerator of LNL with average currents of $\sim 1 \mathrm{pnA}$ onto a $50 \mu \mathrm{g} / \mathrm{cm}^{2}$ ${ }^{40} \mathrm{Ca}$ target. The target was made of $\mathrm{CaF}_{2}$ material, strip (2 $\mathrm{mm}$ width), and supported on a $15 \mu \mathrm{g} / \mathrm{cm}^{2} \mathrm{C}$ backing. The target isotopic enrichment was $\sim 99.8 \%$. ${ }^{42} \mathrm{Ca}$ impurities were at the level of $10^{-5}$ compared to ${ }^{40} \mathrm{Ca}$. The bombarding energy of ALPI has been varied in steps of $15 \mathrm{MeV}$ from 330 to $275 \mathrm{MeV}$. A last energy $\mathrm{E}_{l a b}=255 \mathrm{MeV}$ has been measured with the Tandem XTU only, to have a precise reference (energies are def ned with better than $10^{-3}$ accuracy).

For each ALPI energy measurements have been performed at two additional energies by placing in front of the $\mathrm{Ca}$ targets one and two $\mathrm{C}$-foils with a thickness of 85

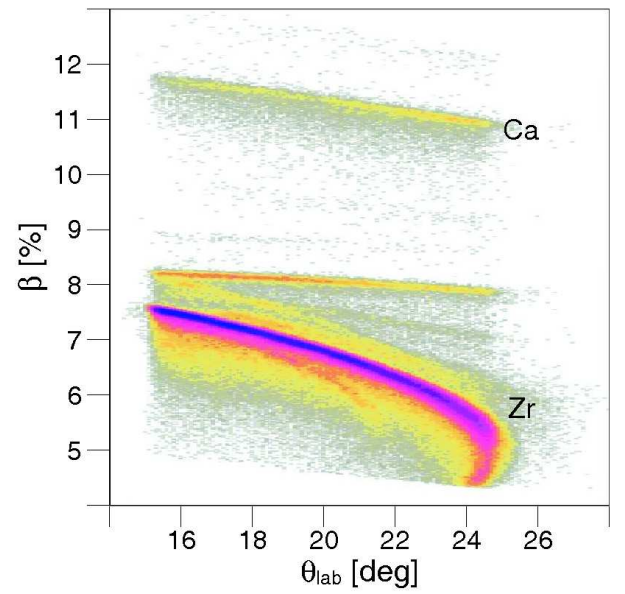

Fig. 1. Example of matrix velocity (quoted as v/c) vs in-plane scattering angle for the reaction ${ }^{96} \mathrm{Zr}+{ }^{40} \mathrm{Ca}$ at $\mathrm{E}_{\text {lab }}=330 \mathrm{MeV}$. Events following a monotonic behaviour correspond to Ca-like recoils $\left(\mathrm{CaF}_{2}\right.$ target). The $\mathrm{Zr}$-like ions (scattered from $\mathrm{Ca}$ as well as from other elements present into the target) and entering into PRISMA follow curved shapes typical of inverse kinematics, with two velocities corresponding to two center of mass angles associated with one laboratory angle.

$\mu \mathrm{g} / \mathrm{cm}^{2}$ each, in such a way to degrade the ${ }^{96} \mathrm{Zr}$ beam by about 6 and $12 \mathrm{MeV}$, respectively. So, a complete excitation function has been measured from the Coulomb barrier $(334 \mathrm{MeV})$ to $\simeq 25 \%$ below, measuring the transfer yields down to $\sim 15.5 \mathrm{fm}$ of distance of closest approach.

Absolute normalization of cross sections and normalizations between different runs have been ensured by two SSBD monitor detectors placed at $\theta_{l a b}=55^{\circ}$ and $\theta_{l a b}=60^{\circ}$ with respect to beam direction and at a distance of $\sim 40$ $\mathrm{cm}$ from the target. The monitors detected the Rutherford scattered Ca-like (as well as ${ }^{19} \mathrm{~F}$ and ${ }^{12} \mathrm{C}$ ) recoils. Ca-like recoils have been detected at $\theta_{l a b}=20^{\circ}$, corresponding to $\theta_{c m} \simeq 140^{\circ}$, with the PRISMA spectrometer. The use of inverse kinematics and the detection at very forward angles, allowed to have, at the same time, enough kinetic energy of the outgoing recoils (for energy and therefore mass resolution) and forward focused angular distribution (high efficiency).

\section{Experimental results}

In Fig. 1 is shown an example of two-dimensional plot velocity (reconstructed via time-of-f ight) vs in-plane scattering angle. One sees the events corresponding to Ca-like recoils as well as the curved shapes corresponding to Zr-like ions entering into PRISMA. In fact, the magnetic f elds set to bring the Ca-ions with maximum yield near the center of the focal plane area, allow part of the $\mathrm{Zr}$ ejectiles to enter into the spectrometer.

Full identif cation in mass (A), nuclear charge (Z) and Q-value of transfer reaction products has been achieved. Nuclear charge $Z$ is obtained through the measurement of energy loss $\Delta E$ and total energy $E$ in the ionization cham- 


\section{FUSION11}

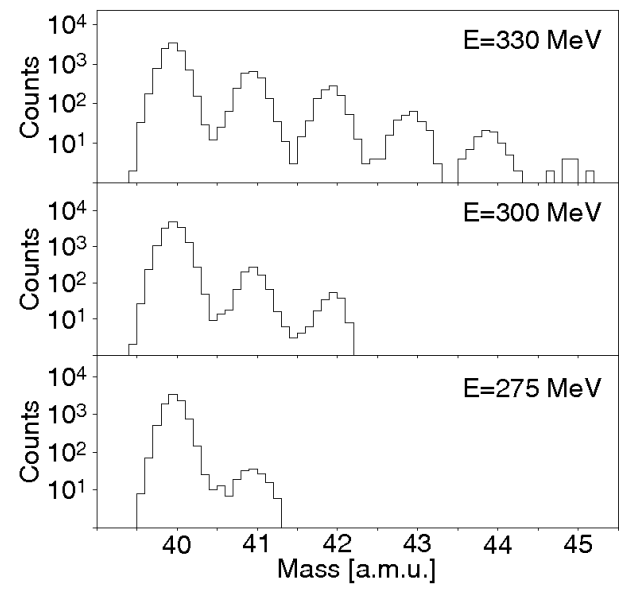

Fig. 2. Mass distributions for pure neutron transfer channels obtained in the reaction ${ }^{96} \mathrm{Zr}+{ }^{40} \mathrm{Ca}$ at the indicated bombarding energies. Ca-like recoils have been detected at $\theta_{l a b}=20^{\circ}$ with the PRISMA spectrometer. The obtained resolution is $\triangle A / A \sim 1 / 150$, which allows to clearly separate the different isotopes.

ber located at the focal plane. Proton transfer yields at subbarrier energies drop off very rapidly thus in this work we discuss pure neutron transfer channels where sufficient statistics has been achieved. Mass identif cation is based on an event-by-event reconstruction of the ion trajectory inside the magnetic elements, through the measurement of entrance (microchannelplate) and exit (multiwire parallelplate) detector positions and time-of-f ight [16]. In the reconstruction procedure a simplifed and fast algorithm has been used, based on the assumption that the trajectory is planar after the quadrupole (longitudinal dimension of PRISMA much larger than the transversal one) and that the fringing felds can be neglected (large dimensions of the magnetic elements).

Mass spectra at representative energies are shown in Fig. 2, where a clear separation of different isotopes is visible. At energies close to the Coulomb barrier we observe the population of more than four neutron pick-up channels, while at sub-barrier energies only one or at most two neutron transfers survive. A signif cant transfer yield could be detected at the level of $10^{-4}$ with respect to the elastic channel. The differential cross sections are obtained taking into account the geometry of monitor and PRISMA detectors and assuming an energy and mass independent ion transmission through the spectrometer. This assumption, validated by a tracing code [17], is well justif ed in the present experiment as we are dealing with pure neutron transfer channels which, at sub-Coulomb energies, have narrow Q-value distributions weakly dependent on isotope and well within the acceptance of the spectrometer.

The spectra of the neutron pick-up channels, whose ground to ground state $\mathrm{Q}$-values are $\mathrm{Q}_{g s}^{+1 n}=+0.14 \mathrm{MeV}$ and $\mathrm{Q}_{g s}^{+2 n}=+5.6 \mathrm{MeV}$, peak at their optimum Q-values, which is close to zero for neutron transfers. The larger Total Kinetic Energy Loss (TKEL) components which are present at above the barrier, ref ecting the onset of deep inelastic processes, disappear below the barrier. Here the distribu- tions get narrower, indicating the damping of excitation energy in the binary partners, and display little dependence both in the position of the centroid and in the shape. ${ }^{42} \mathrm{Ca}$ has been populated via two neutron pick-up channels in ${ }^{40} \mathrm{Ca}+{ }^{208} \mathrm{~Pb}$ [9] and ${ }^{40} \mathrm{Ca}+{ }^{96} \mathrm{Zr}$ [16] reactions at energies slightly above the barrier. The TKEL spectra, peaking at similar values as in the present experiment, leave unpopulated the ground states. This is compatible with the analysis of $\gamma$-particle coincidences [16], which shows that most of the transfer $\mathrm{fux}$ is in the excited states leaving a very small contribution of direct population to the ground to ground states. It has been suggested [18] that the strong population at TKEL $\sim 6 \mathrm{MeV}$ in ${ }^{42} \mathrm{Ca}$ does not only ref ect optimum Q-value arguments but can be an effect of structure.

\section{Transfer probabilities}

Making use of semiclassical conditions, we can extract the transfer probability $\mathrm{P}_{t r}$ as function of the distance of closest approach $\mathrm{D}$, with $\mathrm{P}_{t r}$ def ned as the ratio of transfer cross sections to the elastic one. Such a simplif ed way to phenomenologically describe the trend of cross sections is useful to combine in a single plot values extracted via angular distributions or excitation functions and has been frequently used to compare single and multiple transfers of nucleons [19-21]. This representation is signif cant only if semiclassical conditions are fullf lled and one deals with (almost) pure Coulomb trajectories. The here studied case well fulf lls these requirements, with the further advantage that the Q-value distributions at the measured sub-barrier energies are quite narrow and corresponding to few $\mathrm{MeV}$ of excitation energy. We here remind that slopes smaller than predicted were found for two [22] or more [14] nucleon transfer, where the origin of the discrepancies was attributed to the breakdown of semiclassical conditions [23]. On the contrary, in the few cases where measurements have been performed below the Coulomb barrier no anomaly in the slope behaviour has been clearly identif ed [12,22].

At large ion-ion separation the radial behaviour of the form factor is governed by the exponential form of the bound-state wave function and $\mathrm{P}_{t r}$ is approximated by :

$$
P_{t r}(\theta) \simeq e^{-2 \alpha D(\theta)}
$$

where the parameter $\alpha$ is related to the binding energy $\mathrm{E}_{b}$ of the transferred nucleon, $\alpha=\left(2 m E_{b}\right)^{1 / 2} \hbar$, and $D(\theta)$, for a Coulomb trajectory, is given by:

$$
D(\theta)=\frac{Z_{a} Z_{A} e^{2}}{2 E}\left(1+\frac{1}{\sin \theta / 2}\right) .
$$

The excitation functions (or angular distributions) of transfer processes vs the distance of closest approach D are thus represented (in a semi-logarithmic plot) by straight lines with a slope $-\alpha$. Such behaviour is independent on the way in which transfer proceeds, as a successive process or as a "simultaneous" transfer.

The plot of $\mathrm{P}_{t r}$ is shown in Fig. 3 for the $+1 n,+2 n$ and $+3 n$ transfer channels, together with the solid lines which 


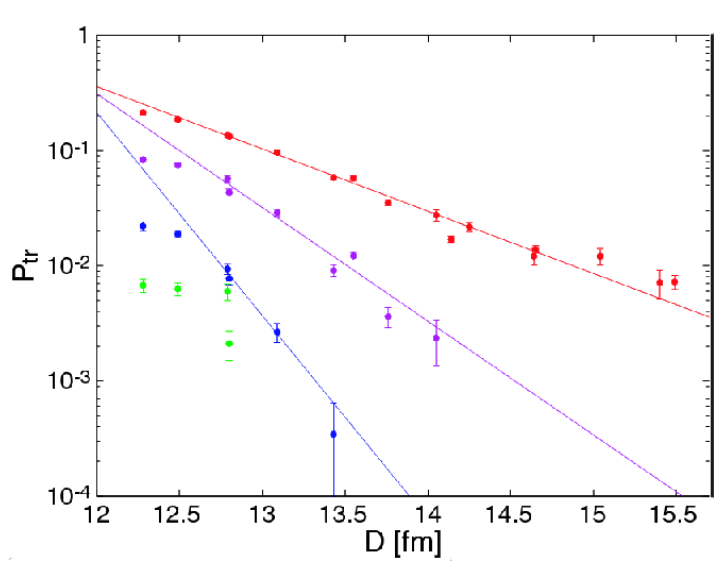

Fig. 3. Transfer probabilities $P_{t r}$ as function of the distance of closest approach D for neutron transfer channels. Points are the experimental data (red: $+1 n$, violet: $+2 n$, blue: $+3 n$, green: $+4 n$ ), lines are $\mathrm{ft}$ with straight lines, according to eq. (1). In the $\mathrm{ft}$ we excluded the points corresponding to the highest measured energies where absorption starts to be signif cant.

are the results of the ftting procedure. Data for the $+4 n$ channel are available only at the highest energies, therefore a reliable $\mathrm{ft}$ could not be performed. The extracted experimental slopes well agree well with those predicted by the binding energies.

Given the correct behaviour of $\mathrm{P}_{t r}$ and keeping in mind the simplif ed assumptions mentioned before, we can make a phenomenological analysis which compares the probabilities for transfer channels with those expected from an independent particle transfer mechanism. It turns out that $P_{t r}^{+2 n} \sim 3 \times\left(P_{t r}^{+1 n}\right)^{2}$ and $P_{t r}^{+3 n} \sim P_{t r}^{+1 n} \times P_{t r}^{+2 n}$. Moreover, even if data for the $+4 \mathrm{n}$ channel could be measured only at the highest energies, the absolute values are consistent with $P_{t r}^{+4 n} \sim\left(P_{t r}^{+2 n}\right)^{2}$. The extracted numbers correspond to slopes which are the average over unresolved excited states of the donor and acceptor nuclei and must be therefore taken with caution, however it turns out that the transfer of even number of neutrons exhibits an enhancement. Whether this ref ects some important physical effect or is simply the result of a very crude estimation cannot be concluded and one has to properly compare the data with theory. A microscopic analysis based on semiclassical theory is discussed in [24].

\section{References}

1. R.A. Broglia and A. Winther, Heavy Ion Reactions (Addison-Wesley Pub. Co., Redwood City CA, 1991).

2. B. F. Bayman and J. Chen, Phys. Rev. C26, (1982) 1509

3. E. Maglione, G. Pollarolo, A. Vitturi, R. A. Broglia and A. Winther, Phys. Lett. B162, (1985) 59

4. L. Corradi, G. Pollarolo and S. Szilner, J. of Phy. G: Nucl. Part. Phys. 36, (2009) 113101

5. A. Winther, Nucl. Phys. A572, (1994) 191

6. A. Winther, Nucl. Phys A594, (1995) 203

7. A. Winther, program GRAZING, www.to.infn.it/ nanni/grazing.
8. L. Corradi et al, Phys. Rev. C66, (2002) 024606

9. S. Szilner, L. Corradi, G. Pollarolo, S. Beghini, B.R. Behera, E. Fioretto, A. Gadea, F. Haas, A. Latina, G. Montagnoli, F. Scarlassara, A.M. Stefanini, M. Trotta, A.M. Vinodkumar, and Y. Wu, Phys. Rev. C71, (2005) 044610

10. C H Dasso and G Pollarolo, Phys. Lett. B155, (1985) 223

11. R. R. Betts, P. M. Evans, C. N. Pass, N. Poffe, A. E. Smith, L. Stuttge, J. S. Lilley, D. W. Banes, K. A. Connell, J. Simpson, J. R. H. Smith, A. N. James, and B. R. Fulton, Phys. Rev. Lett. 59, (1987) 978

12. R. B. Roberts, S. B. Gazes, J. E. Mason, M. Satteson, S. G. Teichmann, L. L. Lee, Jr., L. L. Lee, J. F. Liang, J. C. Mahon, and R. J. Vojtech, Phys. Rev. C47, (1993) R1831

13. C. L. Jiang, K. E. Rehm, J. Gehring, B. Glagola, W. Kutschera, M. Rhein, and A. H. Wuosmaa, Phys. Lett. B337, (1994) 59

14. C. L. Jiang, K. E. Rehm, H. Esbensen, D. J. Blumenthal, B. Crowell, J. Gehring, B. Glagola, J. P. Schiffer, and A. H. Wuosmaa, Phys. Rev. C57, (1998) 2393

15. A. M. Stefanini, L. Corradi, G. Maron, A. Pisent, M. Trotta, A. M. Vinodkumar, S. Beghini, G. Montagnoli, F. Scarlassara, G. F. Segato, A. De Rosa, G. Inglima, D. Pierroutsakou, M. Romoli, M. Sandoli, G. Pollarolo, and A. Latina, Nucl. Phys. A701, (2002) 217c

16. S. Szilner, C. A. Ur, L. Corradi, N. Marginean, G. Pollarolo, A. M. Stefanini, S. Beghini, B. R. Behera, E. Fioretto, A. Gadea, B. Guiot, A. Latina, P. Mason, G. Montagnoli, F. Scarlassara, M. Trotta, G. de Angelis, F. Della Vedova, E. Farnea, F. Haas, S. Lenzi, S. Lunardi, R. Marginean, R. Menegazzo, D. R. Napoli, M. Nespolo, I. V. Pokrovsky, F. Recchia, M. Romoli, M.-D. Salsac, N. Soic, and J. J. Valiente-Doóbon, Phys. Rev. C76, (2007) 024604

17. D. Montanari, E. Farnea, S. Leoni, G. Pollarolo, L. Corradi, G. Benzoni, A. Gadea, E. Fioretto, A. Latina, G. Montagnoli, F. Scarlassara, A.M. Stefanini and S.Szilner, Eur. Phys. J. A 47, (2011) 4

18. S. Szilner, L. Corradi, F. Haas, G. Pollarolo, S. Beghini, B.R. Behera, E. Caurier, E. Fioretto, A. Gadea, A. Latina, G. Montagnoli, F. Nowacki, F. Scarlassara, A.M. Stefanini, M. Trotta, A.M. Vinodkumar, and Y.W. Wu, Eur. Phys. J. A21, (2004) 87

19. W. von Oertzen, H. G. Bohlen, B. Gebauer, R. Kunkel, F. Puhlhofer, and D. Schull, Z. Phys. A326, (1987) 463

20. W. von Oertzen, I. Peter, S. Thummerer, H. G. Bohlen, B. Gebauer, J. Gerl, M. Kaspar, I. Kozhoukharov, T. Kroll, M. Rejmund, and H. J. Wollersheim, Eur. Phys. J. A20, (2004) 153

21. W.von Oertzen and A.Vitturi, Rep. Prog. Phys. 64, (2001) 1247

22. K.E.Rehm, B.G.Glagola, W.Kutschera, F.L.H.Wolfs, H.Wuosmaa, Phys. Rev. C47, (1993) 2731

23. A.H. Wuosmaa, K.E. Rehm, B.G. Glagola, Th. Happ, W. Kutschera, and F.L.H. Wolfs, Phys. Lett. B255, (1991) 316

24. G. Pollarolo, contribution to this Conference 